\title{
Intensity-dependent effect of treadmill running on lubricin metabolism of rat articular cartilage
}

\author{
Guo-Xin $\mathrm{Ni}^{1 *}$, Lei Lei ${ }^{2}$ and Yue-Zhu Zhou ${ }^{3}$
}

\begin{abstract}
Introduction: We aimed to understand the changes in cartilage lubricin expression and immunolocalisation in responsed to treadmill running with different intensities in a rat model.

Methods: A total of 24 male Wistar rats were randomly assigned into groups of control (CON), low-intensity running (LIR), moderate-intensity running (MIR), and high-intensity running (HIR). Rats in LIR, MIR, and HIR groups were trained for 8 weeks on the treadmill with low, moderate, and high intensity, respectively. After sacrifice, femoral condyles were collected to take histological observation for cartilage characteristics, and immunohistochemistry for lubricin. In addition, cartilage samples were obtained to assess PRG4 and TGF- $\beta$ mRNA expression by quantitative RT-PCR.
\end{abstract}

Results: Histological examination showed osteoarthritic changes in rats after eight weeks of high intensity running. In comparison to CON group, significantly lower Mankin score was found in LIR and MIR groups, whereas, HIR group had significantly higher Mankin score than either CON, LIR, or MIR group. On the other hand, both LIR and MIR groups have significantly higher lubricin content than CON group, whereas, significantly lower lubricin content was found in HIR group compared with CON, LIR or MIR group. A significant inverse correlation was detected between the lubricin content and Mankin score. In addition, considerably higher mRNA gene expression of PRG4 and TGF- $\beta$ was found in LIR and MIR groups, compared with those in CON and HIR groups.

Conclusions: There is a marked intensity-specific effect of running on the immunolocalisation and gene expression of lubricin in cartilage, which is inversely correlated with Mankin score. Our findings provide evidences that mechanical factors are key determinants of lubricin metabolism in vivo.

\section{Introduction}

In addition to transmitting load, another major function of articular cartilage is to provide a low-friction surface that allows the bones of diarthrodial joints to slide smoothly against each other. Such remarkable frictional properties of the tissue are achieved, at least in part, by lubricin, a mucinous glycoprotein synthesized and secreted into synovial fluid both by chondrocytes in the superficial zone of articular cartilage [1] and by synoviocytes [2], and which is encoded by the proteoglycan 4 (PRG4) gene [3]. There are a number of post translational products of the PRG4 gene [2-6], including megakaryocyte stimulating factor precursor and superficial zone protein (SZP). In this

\footnotetext{
* Correspondence: fgxni@graduate.hku.hk

'Department of Orthopaedics and Traumatology, Nanfang Hospital, Southern Medical University, 1838 Guangzhou Avenue (N), Guangzhou 510515, China
} Full list of author information is available at the end of the article article, these homologous proteins are referred to as lubricin.

While functioning as a boundary lubricant in articular joints, lubricin is also recognized to have a major protective role in preventing cartilage wear, and synovial cell adhesion and proliferation [7]. Obviously, lubricin plays an important role in articular joint physiology, and the loss of accumulation of lubricin may have a role in the pathology of osteoarthritis (OA). Indeed, lubricin synthesis is downregulated in a number of animal models of OA [8,9]. In addition, in mice lacking the lubricin gene, there is alteration of the articular surface and attendant degradation of articular cartilage, causing early-onset noninflammatory joint damage and failure [10]. Furthermore, a recent study by Flannery et al. [11] demonstrated that intra-articular lubricin injection following an anterior cruciate ligament (ACL) injury was beneficial in retarding the degeneration of cartilage and the development of post-traumatic OA.

\section{C) Biomed Central}


In vitro evidences suggest that mechanical stimulation can affect the biosynthesis and secretion of lubricin. Nugent et al. $[12,13]$ found that both static and dynamic shear stimulation applied to cartilage explants altered cartilage secretion of lubricin relative to that of unloaded controls. More recently, using a bioreactor, Nugent-Derfus et al. [14] suggested that lubricin secretion rate varied markedly over the joint surface following 24 hours of continuous passive motion (CPM). Nevertheless, as the load experienced by natural joints is more complicated than simple compression, shear or pressurization, it is difficult to extrapolate these in vitro results to the in vivo situation.

Running is one of the most common weight-bearing activities. However, the in vivo regulatory effect of running load on the biosynthesis of lubricin remains unknown. For one thing, marked magnitude-dependent regulatory effects of mechanical stimuli have been suggested in vitro on cartilage biosynthesis of lubricin molecules $[12,13]$. On the other hand, the homeostasis of cartilage was stabilized in a physiological range of mechanical load, non-physiological mechanical load, both overload and reduced load, may have deleterious effects [15-17]. Specifically, moderate running exercise was found to protect against cartilage degradation in hamsters that had spontaneously developed OA [18]. However, excessive running load has been correlated with deleterious effects on knee cartilage in rats that did not spontaneously suffer from OA [19-21]. A recent study reported that there was a significant inverse correlation between the lubricin concentration in synovial fluid and the Mankin score of guinea pig knee cartilage [22]. It is therefore hypothesized that the running-induced load may regulate in vivo biosynthesis of lubricin with intensity dependence. To assess this hypothesis, the current study was undertaken to determine the changes in cartilage lubricin expression and immunolocalisation in response to treadmill running with different intensities on the knee joint of rats.

\section{Materials and methods}

\section{Experimental animals and exercise protocols}

A total of 24 male Wistar rats (12 to 13 week old, weight 200 to $250 \mathrm{~g}$ ) were randomly and evenly assigned to one of four groups as follows: 1) sedentary control (CON, $n=6)$; 2) low intensity running (LIR, $n=6$ ); 3 ) moderate intensity running (MIR, $n=6$ ); and 4 ) high intensity running (HIR, $n=6$ ). Rats were housed in cages under controlled light/dark $(12 / 12 \mathrm{~h})$ and temperature $\left(22 \pm 1^{\circ} \mathrm{C}\right)$ conditions, and were provided with food and water ad libitum. They were adapted to laboratory conditions for 1 week before experiments began. This study was approved by the animal ethics committee of the institute.

All rats were firstly accustomed to exercise for 1 week, by running on a treadmill at a speed of $10 \mathrm{~m} / \mathrm{min}$ for
30 minutes/day. Subsequently, animals in the LIR, MIR, and HIR groups were regularly trained according to the running protocol (Table 1) for 8 weeks, to elicit the low, moderate, and high intensity exercise respectively, for the Wistar rats, [23], while the CON group served as the control. All experiments were conducted in accordance with our institutional guidelines for the care and use of experimental animals.

\section{Tissue preparation}

For histological morphology and immunohistochemistry examinations, femoral condyles of the medial compartment on the right sides of rats in each group were dissected and fixed in $4 \%$ buffered formaldehyde $\mathrm{pH} 7.4$ for 24 hours. Decalcification was completed in $10 \%$ ethylenediaminetetraacetic acid (EDTA) solution, and then the samples were embedded in paraffin wax. Thereafter, they were cut into 5 -mm sagittal sections in the medial region. In each section, three areas (non-contact, transitional and contact areas) were chosen for histomorphological evaluation and immunohistological analysis (Figure 1).

For quantitative real-time PCR analysis, articular cartilage samples from femoral condyles on the left sides were obtained with a scalpel or rongeur, and flash-frozen in liquid nitrogen at $-80^{\circ} \mathrm{C}$.

\section{Histomorphological evaluation}

The samples were stained with Safranin-O and histomorphologically evaluated with the modified Mankin scoring system, which has previously been applied to many experimental OA models $[18,19]$, and proven to be sensitive to early OA changes induced by treadmill exercise [24]. All sections were graded by two independent observers (LL and YZZ) who were unaware of the grouping, and the mean score was calculated for each area in each section.

\section{Immunohistochemistry for lubricin}

In addition to histomorphological evaluation, immunohistological analysis for lubricin was performed in the above-mentioned three areas of each section. After deparaffinization and rehydration of the tissue sections, lubricin was immunostained with the two-step immunohistochemistry method as instructed by the manufacturer.

The sections were incubated overnight with rabbit polyclonal antibody against rat lubricin (sc-98454) (Santa Cruz Biotechnology INC., Santa Cruz, CA, USA),

\section{Table 1 Treadmill running protocols for rats}

\begin{tabular}{lllll}
\hline Group & Speed & Inclination & Duration & Frequency \\
\hline LIR & $15.2 \mathrm{~m} /$ minute & $0^{\circ}$ & 60 minutes & 5 days/week \\
MIR & $19.3 \mathrm{~m} /$ minute & $5^{\circ}$ & 60 minutes & 5 days/week \\
HIR & $26.8 \mathrm{~m} /$ minute & $10^{\circ}$ & 60 minutes & 5 days/week \\
\hline
\end{tabular}

LIR, MIR and HIR, low-, moderate- and high-intensity running groups. 


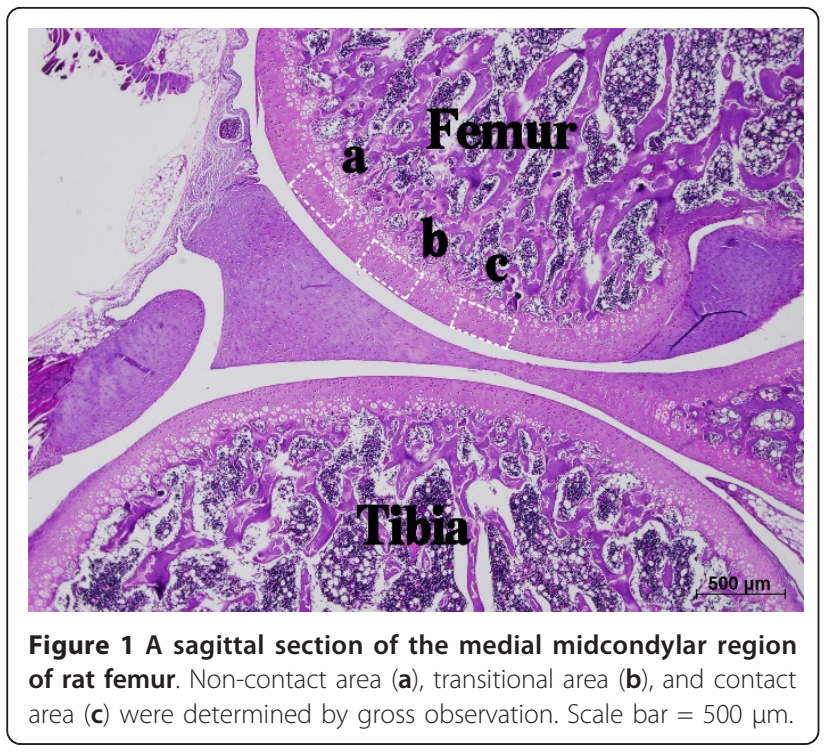

1:200 dilution, at $4^{\circ} \mathrm{C}$. Its epitope corresponds to amino acids 1265-1404 mapping at the C-terminus of Lucricin of human origin. The slides were washed three times in PBS followed by a 30-minute incubation at room temperature with goat anti-rabbit immunoglobulin G (IgG) (Santa Cruz Biotechnology INC., Santa Cruz, CA, USA) and visualized with $\mathrm{DAB}$ chromagen. The slides were stained for $40 \mathrm{sec}$, and then the nucleus was counterstained with hematoxylin for $6 \mathrm{sec}$. Negative control sections were prepared using the same protocol described above, but primary antibody was replaced by PBS. All of the sections were stained concomitantly, at the same time by the same person (YZZ). For each section, three regions were digitally captured with a color video camera attached to a light microscope (Nikon H600L Microscope and image analysis system, Tokyo, Japan), at 400× magnification with constant illumination intensity. Images were captured using Image-Pro Plus software (Media Cybernetics, Silver Spring, MD, USA) with predetermined threshold parameters for lubricin staining. Areas of interest (AOI) from surfaces to the subchondral of the femoral condyle cartilage were selected using the irregular AOI tools. The IOD (integrated optical density) and area were calculated for each region using Image-Pro Plus 6.0 software. The ratio of IOD to area in each region was obtained, and subsequently averaged for lubricin content in each section.
Quantitative real-time polymerase chain reaction (RT-PCR)

The cartilage samples were frozen in liquid nitrogen, and then were broken into pieces with a masher. Total RNA was prepared using the Trizol reagent (Invitrogen Life Technologies, Carlsbad, CA, USA) according to the manufacturer's instructions. RT-PCR was performed on $0.5 \mathrm{mg}$ total RNA using a PrimeScript RT reagent kit with gDNA Eraser (Takara Biotechnology (Dalian) Co. LTD., Dalian, China). Quantitative PCR was performed using an ABI 7500 Real-Time PCR system and a QuantiTect SYBR Green PCR (Takara Biotechnology (Dalian) Co. LTD., Dalian, China); glyceraldehyde-3-phosphate dehydrogenase $(G A P D H)$ was used as an endogenous reference, and each sample was normalized to its GAPDH content. All experiments were performed in duplicate and repeated twice. Primers for quantitative PCR are shown in Table 2.

\section{Statistical analysis}

Results are expressed as the mean \pm SD. Statistical analysis was carried out using one-way analysis of variance (ANOVA) and Tukey's test for post hoc analysis with significance set at $P<0.05$. The relationship between the Mankin score versus the lubricin level was assessed with regression analysis.

\section{Results}

\section{Histological observation}

Figure 2 shows the histological features of femoral articular cartilage with safranin-O staining at contact, transitional, and non-contact areas in the four groups. Similar to the CON group, grossly normal histological characteristics of cartilage sections were observed in the LIR and MIR groups. However, it appears that the increased staining for safranin $\mathrm{O}$ was in the LIR and MIR groups in comparison with CON group. Within the CON, LIR and MIR groups, no distinct differences were found among the contact, transitional, and noncontact areas. In contrast, osteoarthritic histological changes of surface irregularities, cell cloning, and marked reduction in the safranin-O staining were found in the HIR group, with the most severe changes at the contact area.

Histomorphological evaluation was made by the modified Mankin scoring system at contact, transitional, and non-contact areas in the four groups. A similar pattern of changes was observed for each area as well as for the

Table 2 Primer sequence used in quantitative PCR

\begin{tabular}{lll}
\hline Primer & Forward & Reverse \\
\hline GAPDH & 5'-GGCACAGTCAAGGCTGAGAATG -3' & 5'-ATGGTGGTGAAGACGCCAGTA-3' \\
PRG4 & 5'- AGGGCGTGGATCCAAGAA -3' & 5'-ACAGTTGCAGGTGGCGTCTCTA-3' \\
TGF- $\beta 1$ & 5'- TGCGCCTGCAGAGATTCAAG -3' & 5'- TAACGCCAGGAATTGTGCTA-3' \\
\hline
\end{tabular}

GAPDH, glyceraldehyde-3-phosphate dehydrogenase; PRG4, proteoglycan; TGF- $\beta 1$, transforming growth factor- $\beta 1$. 


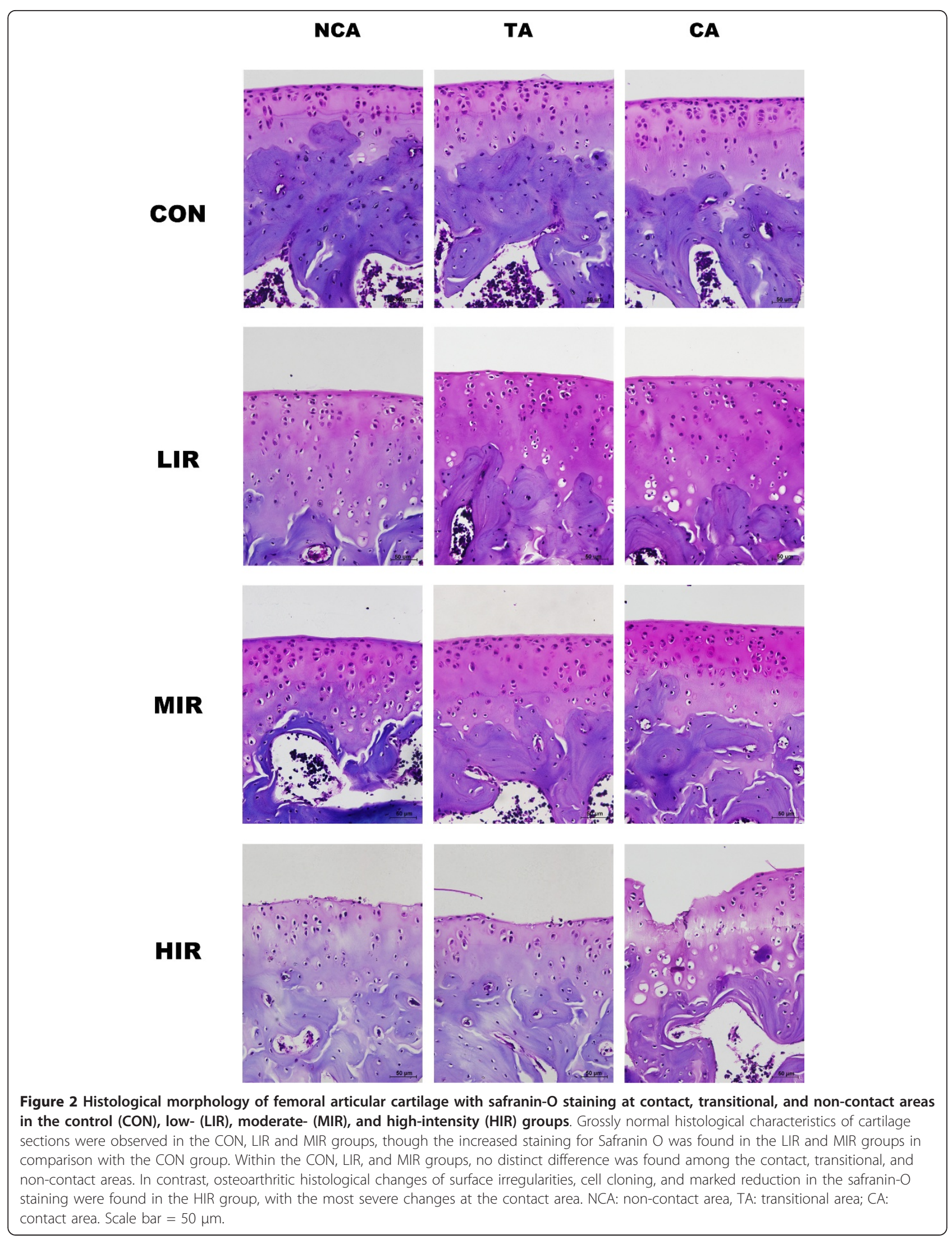


average (Figure 3): both the LIR and MIR groups had lower Mankin score than the CON group. However, a significantly higher Mankin score was found in the HIR group compared to the CON, LIR, or MIR groups, respectively. No statistical difference was found between the LIR and MIR group.

\section{Immunohistochemistry of lubricin}

Figure 4 shows the immunostaining of lubricin in cartilage sections at the three areas in the four groups. Lubricin was mainly immunolocalised to chondrocytes and extracellular matrix (ECM) in the superficial zone of normal cartilage. Markedly increased staining of lubricin was found in the LIR and MIR groups, whereas little staining was detected in the HIR group.

Figure 5 indicates the lubricin content at the contact, transitional, and non-contact areas in the four groups. For each group, the lubricin content for the three areas was averaged. Among the four groups, a similar pattern of changes was observed for area as well as the average. Both the LIR and MIR groups had higher lubricin content than the CON group with statistical significance only for the average. However, significantly lower lubricin content was found in the HIR group, compared to the LIR and MIR groups at the three areas. On average, a statistically significant difference was found between the CON and HIR groups. No statistical difference was found between the LIR and MIR groups. In addition, a significant inverse correlation $(P<0.01)$ was found between the lubricin content

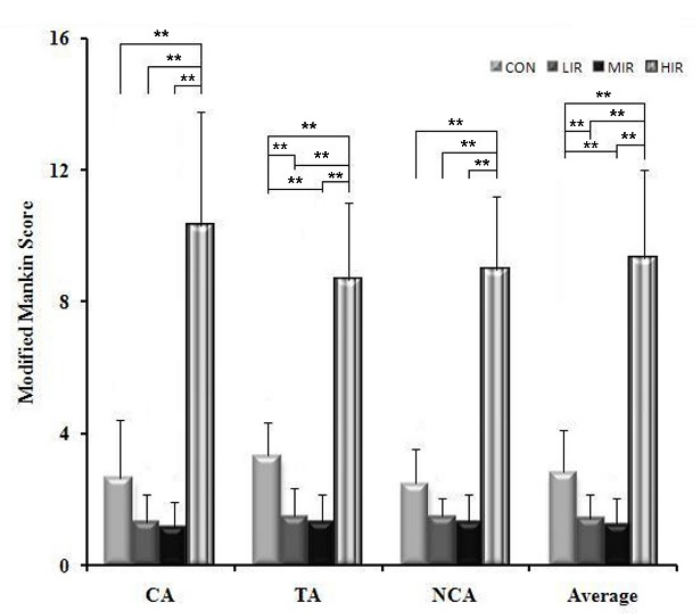

Figure 3 Mankin score at the contact, transitional, and noncontact areas and average for all three areas in the control (CON), low- (LIR), moderate- (MIR), and high-intensity (HIR) groups. Similar changes were observed for each area as well as the average: both the LIR and MIR groups had a lower Mankin score than the CON group, and a significantly higher score was found in the HIR compared to the CON, LIR, and MIR groups, respectively. No statistical difference was found between the LIR and MIR groups. NCA: non-contact area, TA: transitional area; CA: contact area. and the Mankin score either in each group or as a whole (Table 3).

\section{Real-time quantitative PCR for PRG4 and transforming growth factor (TGF)- $\beta$}

As shown in Figure 6A, there was a statistically significant increase of mRNA gene expression of PRG4 in the LIR $(1.03 \pm 0.38)$ and MIR groups $(1.00 \pm 0.12)$ than in the CON group $(0.67 \pm 0.31)$. However, $P R G 4$ expression was significantly lower in the HIR group $(0.64 \pm 0.09)$ than that in the LIR or MIR groups. A similar pattern of changes was found in mRNA gene expression of TGF- $\beta$. The LIR $(1.13 \pm 0.35)$ and MIR $(0.89 \pm 0.09)$ groups had significantly higher gene expression than the CON group $(0.65 \pm 0.20)$, whereas, the HIR group had significantly lower expression $(0.48 \pm 0.08)$ than the LIR or MIR groups (Figure 6B).

\section{Discussion}

It is well recognized that exercise and joint loading can alter articular cartilage composition through alterations in chondrocyte metabolism [25]. Nevertheless, their effects on cartilage lubricin, a mucinous glycoprotein synthesized by chondrocytes in the superficial zone of articular cartilage [1], remain poorly understood. To our knowledge this is the first report describing the immunolocalisation and gene expression of cartilage in response to treadmill running. Our results suggest a marked intensity-specific effect of running on the immunolocalisation and gene expression of lubricin in cartilage. In addition, a similar running-intensity-dependent effect was found on the gene expression of TGF- $\beta$.

Previously, biomechanical regulation of metabolism of lubricin by chondrocytes was well documented in vitro [11-14]. As suggested by the current study, runninginduced joint loading has significant effects on the in vivo synthesis of lubricin, and such effects vary with running intensity. What is more, a significant inverse correlation between the lubricin level and Mankin score is demonstrated, indicating that lubricin might be partially responsible for the change in cartilage. Low- or moderate-intensity running is associated with up-regulation of $P R G 4$, leading to the increase in secretion and synthesis of lubricin. It is well accepted that lubricin plays an important role in articular joint physiology, because it can provide an important property for normal joint function by conferring wear resistance in addition to boundary lubrication. Therefore, low- to moderate-intensity running should have beneficial effect on the health of normal cartilage by up-regulating the biosynthesis of lubricin. On the other hand, exercise is one of the most widely prescribed nonpharmacological therapies for OA management. However, the mechanism underlying its benefit is largely unknown [26]. Galois et al. [27] reported that moderate-intensity running can 


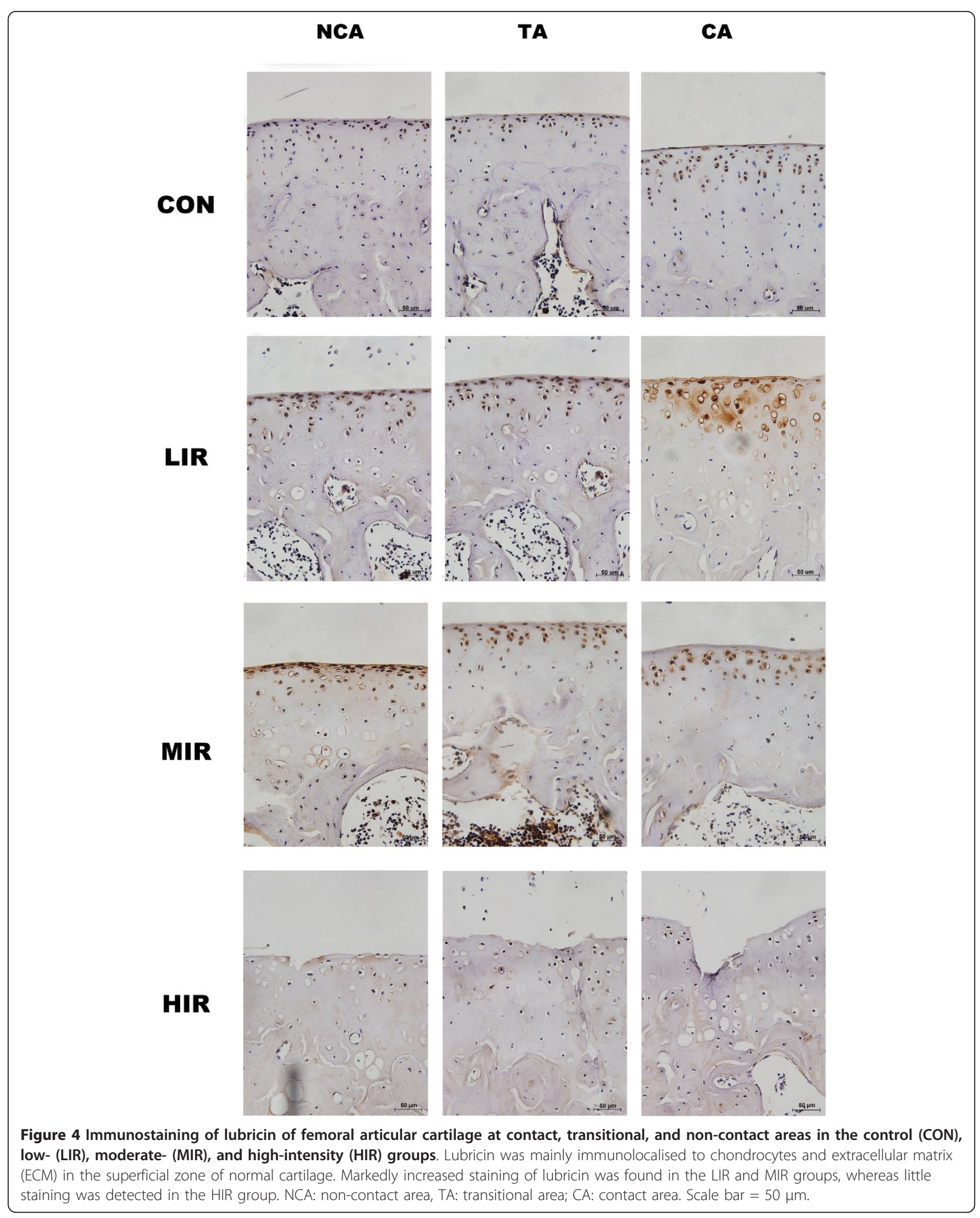




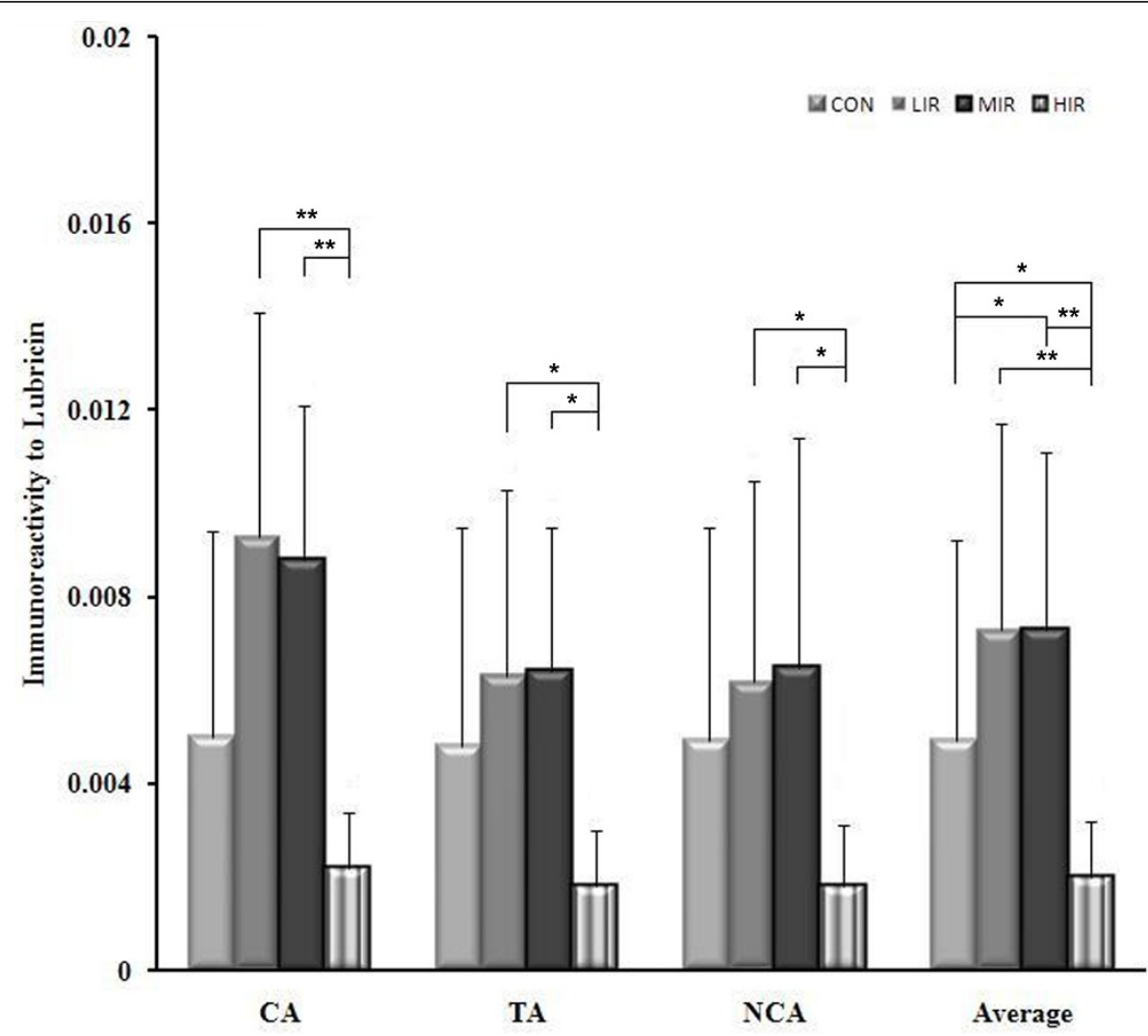

Figure 5 Lubricin content at the contact, transitional, and non-contact areas and average for all three areas in the control (CON), low(LIR), moderate- (MIR), and high-intensity (HIR) groups. Similar changes were observed for each area as well as the average: both the LIR and MIR groups had higher lubricin content than the CON group, and significantly lower lubricin content was found in the HIR group compared to the LIR and MIR groups. No statistical difference was found between the LIR and MIR groups. NCA: non-contact area, TA: transitional area; CA: contact area.

decrease severity of experimental OA in rats. Although further investigations are needed, it is supposed that appropriate exercise can protect against increased cartilage damage by increasing the secretion and synthesis of lubricin in degenerative cartilage. Substantial evidence has been provided on the role of intra-articular lubricin administration in the prevention of post-traumatic OA [11,28-30].

Conversely, our results showed that excessive runninginduced load leads to decreased lubricin level and gene expression. Recently, Elsaid et al. [30] reported that forced joint exercise resulted in decreased lubricin cartilage expression, increased cartilage degeneration and reduced superficial zone chondrocyte viability in the ACLT (anterior cruciate ligament transaction) joint. Using a meniscectomy-induced (that is, mechanically induced) OA model in sheep, Young et al. [9] reported that abnormal joint motion resulted in degeneration of articular cartilage in certain regions of the tibial plateau, where lubricin gene expression decreased. A number of previous studies, including ours [19-21,24], have shown that excessive load has a detrimental effect on cartilage. Such detrimental effect is quite likely to be associated with the decrease in lubricin level due to the significant inverse correlation between the lubricin level and Mankin score indicated in the current study. Coles et al. [31] found that the deletion of lubricin resulted in significant structural and biomechanical changes in the articular cartilage with age, again, suggesting a significant role of lubricin in preserving normal joint structure and function. A recent study showed that a mouse with only one functional allele for lubricin did not

Table 3 Correlation between lubricin content and Mankin score

\begin{tabular}{ccccc}
\hline \multicolumn{3}{c}{ Group } & & Total $(n=72)$ \\
\hline $\operatorname{CON}(n=18)$ & $\operatorname{LIR}(n=18)$ & MIR $(n=18)$ & HIR $(\boldsymbol{n}=18)$ & $0.42^{* *}$ \\
\hline $0.593^{* *}$ & $0.692^{* *}$ & $0.76^{* *}$ & $0.816^{* *}$ & \\
\hline
\end{tabular}

CON, control group; LIR, MIR and HIR, low-, moderate- and high-intensity running groups. * ${ }^{*}$ Significantly different from zero $P<0.01$. 


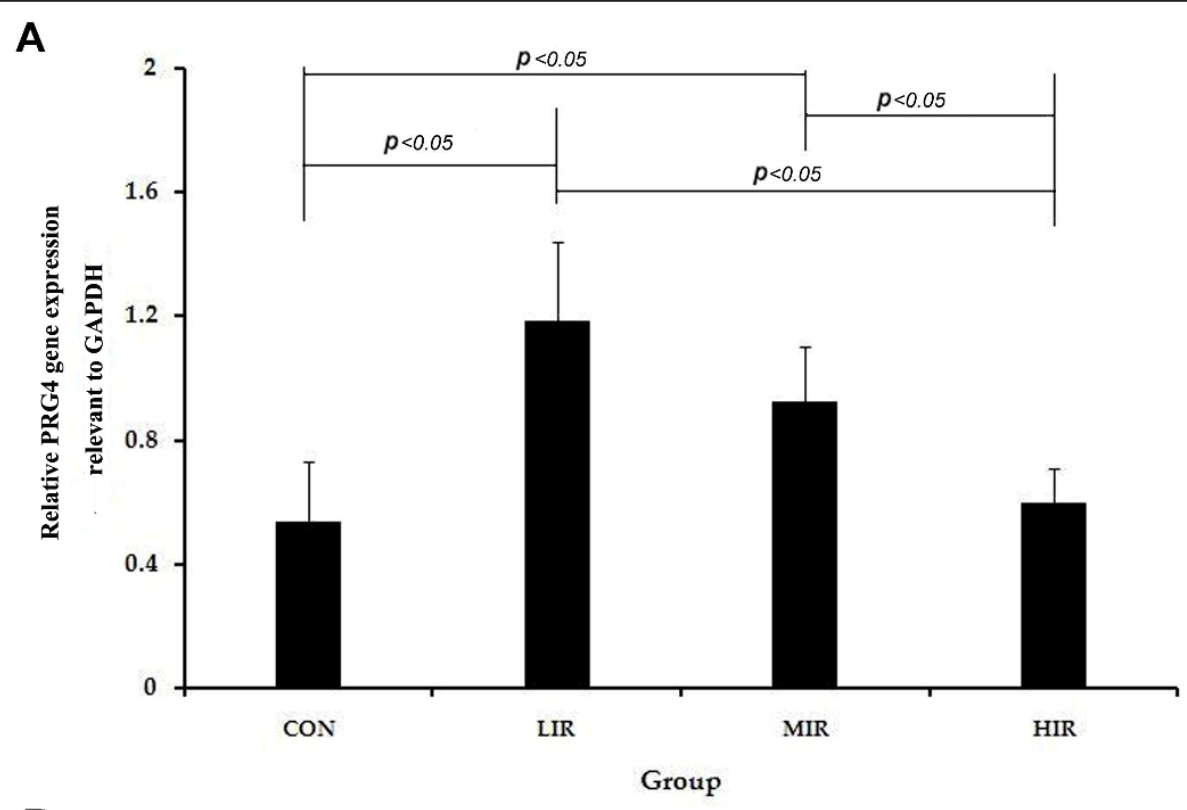

B

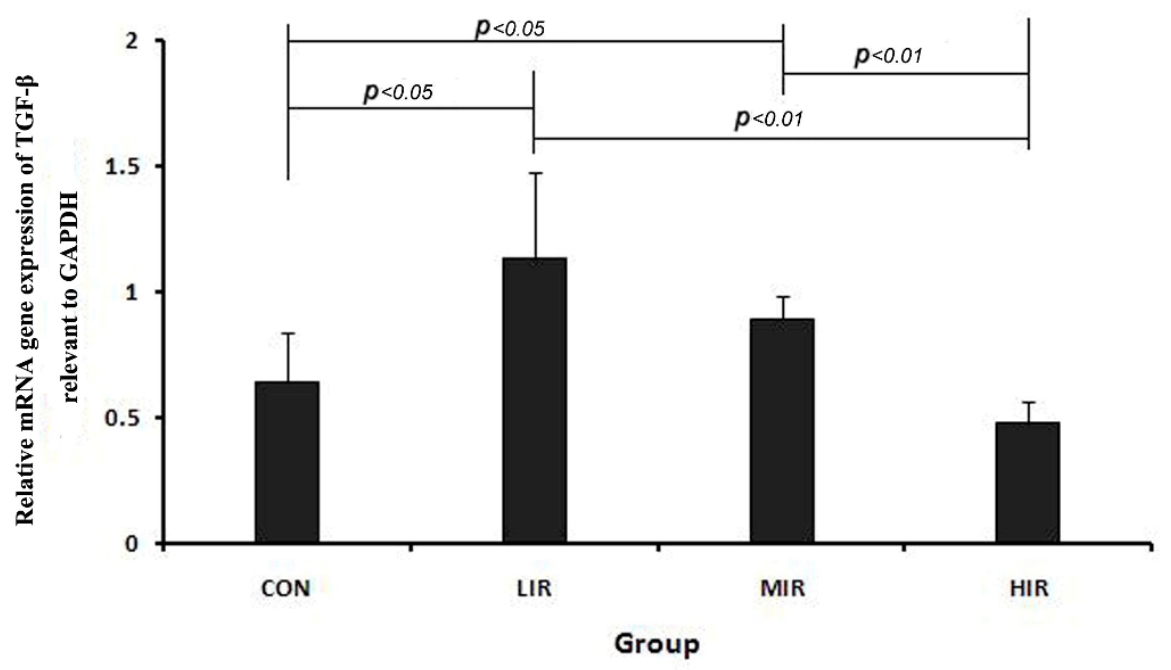

Figure 6 Relative mRNA gene expression of proteoglycan (PRG)4 (A) and transforming growth factor (TGF)- $\beta$ (B) relevant to lyceraldehydes-3-phosphate dehydrogenase (GAPDH) in the control (CON), low- (LIR), moderate- (MIR), and high-intensity (HIR) groups. Significantly higher mRNA gene expression of PRG4 was found in the LIR (1.03 \pm 0.38$)$ and MIR group (1.00 \pm 0.12$)$ than in the CON group $(0.67 \pm 0.31)$. However, there was significantly lower PRG4 expression in the HIR group $(0.64 \pm 0.09)$ than in LIR or MIR groups. Similar changes were found in mRNA gene expression of TGF- $\beta$. The LIR (1.13 \pm 0.35$)$ and MIR $(0.89 \pm 0.09)$ groups had significantly higher gene expression than the CON group $(0.65 \pm 0.20)$, whereas the HIR group $(0.48 \pm 0.08)$ had significantly lower expression than the LIR or MIR groups. NCA: non-contact area, TA: transitional area; CA: contact area.

provide chondroprotection ex vivo as its joint was loaded and oscillated [32], which is consistent with our finding that, under condition of excessive wear, lubricin as a sacrificial boundary layer may succumb to overuse. Taken together, it is believed that the down-regulation of lubricin by excessive running load, at least partially, contributes to the cartilage damage.
Similarly to previous reports of lubricin immunolocalisation in normal cartilage [5,9], lubricin-positive cells and matrix were observed mainly in the superficial zone in the current study. However, site-associated variation was detected between contact, non-contact, and transitional regions. Similar results were previously reported by Nugent-Derfus et al. [14]. Such variation was thought to 
be associated with the apparent dependence on the local mechanical environment in different regions over the surface of the cartilage [33-35]. Taken together with the altered lubricin level and gene expression patterns subjected to different running-induced loads, this investigation further supports the hypothesis that mechanical factors are key determinants of lubricin metabolism in vivo.

Interestingly, under different mechanical conditions (that is, low-, moderate-, and high-intensity running load), a synchronous changing pattern was found for lubricin levels in three different areas of the cartilage surface. For each area, the lubricin level increased under a low- or moderate-running-induced load, and decreased under excessive running load. This implies a corresponding safe upper limit of stress for each area. As the corresponding stress acting on different areas differs, the stress threshold should not be identical in these areas. According to the so-called conditioning hypothesis proposed by Seedhom [36], the stress threshold of cartilage is regulated by the prevalent stresses arising in joints. We therefore postulate that the contact region has a higher stress threshold than the other regions due to its relatively higher prevalent stress.

The mechanisms involved in regulating lubricin gene expression and synthesis remain largely unknown. In the current study, similar to PRG4, the expression of TGF- $\beta$ was up-regulated under a low- and moderate-running load, but down-regulated under excessive running load. Neu et al. [35] suggested that mechanotransduction of lubricin expression in articular cartilage occurred through TGF- $\beta$ mediated signaling pathways. A body of evidence has showed that TGF- $\beta$ stimulates lubricin synthesis [37-40], and thus may be beneficial for normal cartilage function $[2,41]$. It is therefore assumed that the regulation of running-induced load on the biosynthesis of lubricin is through the TGF- $\beta$ mediated signaling pathway, or a combination with the direct mechanical regulation. Future work will be necessary to understand the precise mechanisms.

\section{Conclusions}

In the current study, a marked intensity-specific effect of running on the immunolocalisation and gene expression of lubricin in cartilage is demonstrated. In addition, the significant inverse correlation between the lubricin level and the Mankin score indicates that lubricin might be partially responsible for the change in cartilage. Our findings provide evidence that mechanical factors are key determinants of lubricin metabolism in vivo.

\section{Abbreviations}

ACL: anterior cruciate ligament; ANOVA: analysis of variance; $A O I$ : areas of interest; CPM: continuous passive motion; ECM: extracellular matrix; HIR: high intensity running; IgG: immunoglobulin G; IOD: integrated optical density; LIR: low intensity running; MIR: moderate intensity running; OA: osteoarthritis; PBS: phosphate-buffered saline; RT-PCR: real-time polymerase chain reaction; PRG4: proteoglycan 4; SZP: superficial zone protein; TGF: transforming growth factor.

\section{Acknowledgements}

We gratefully acknowledge Mr PR Zhao for technical assistance. This work was supported by special funding for university talent introduction of Guangdong Province (GX N).

\section{Author details}

${ }^{1}$ Department of Orthopaedics and Traumatology, Nanfang Hospital, Southern Medical University, 1838 Guangzhou Avenue (N), Guangzhou 510515, China. 2Department of Rehabilitation Medicine, Longyan People's Hospital, 31 Denggao Road (W), Longyan 364000, China. ${ }^{3}$ Department of Rehabilitation, $1^{\text {st }}$ Affiliated Hospital, Fujian Medical University, 20 Chazhong Road, Fuzhou 350005, China.

\section{Authors' contributions}

GN conceived the study, participated in the design, and wrote most of the manuscript. $L L$ and $Y Z$ performed the experiments, analyzed data and helped to draft the manuscript. All authors read and approved the final manuscript.

\section{Competing interests}

The authors declare that they have no competing interests.

Received: 15 June 2012 Revised: 31 October 2012

Accepted: 22 November 2012 Published: 24 November 2012

\section{References}

1. Su JL, Schumacher BL, Lindley KM, Soloveychik V, Burkhart W, Triantafillou JA, Kuettner K, Schmid T: Detection of superficial zone protein in human and animal body fluids by cross-species monoclonal antibodies specific to superficial zone protein. Hybridoma 2001, 20:149-157.

2. Schumacher BL, Hughes CE, Kuettner KE, Caterson B, Aydelotte MB: Immunodetection and partial CDNA sequence of the proteoglycan, superficial zone protein, synthesized by cells lining synovial joints. J Orthop Res 1999, 17:110-120.

3. Ikegawa S, Sano M, Koshizuka Y, Nakamura Y: Isolation, characterization and mapping of the mouse and human PRG4 (proteoglycan 4) genes. Cytogenet Cell Genet 2000, 90:291-297.

4. Marcelino J, Carpten JD, Suwairi WM, Gutierrez OM, Schwartz S, Robbins C, Sood R, Makalowska I, Baxevanis A, Johnstone B, Laxer RM, Zemel L, Kim CA, Herd JK, Ihle J, Williams C, Johnson M, Raman V, Alonso LG, Brunoni D, Gerstein A, Papadopoulos N, Bahabri SA, Trent JM, Warman ML: $C A C P$, encoding a secreted proteoglycan, is mutated in camptodactylyarthropathy-coxa vara-pericarditis syndrome. Nature Genet 1999, 23:319-322.

5. Jay GD, Britt DE, Cha CJ: Lubricin is a product of megakaryocyte stimulating factor gene expression by human synovial fibroblasts. J Rheumatol 2000, 27:594-600.

6. Jay GD, Tantravahi U, Britt DE, Barrach HJ, Cha CJ: Homology of lubricin and superficial zone protein (SZP): products of megakaryocyte stimulating factor (MSF) gene expression by human synovial fibroblasts and articular chondrocytes localized to chromosome 1q25. J Orthop Res 2001, 19:677-687.

7. Rhee DK, Marcelino J, Baker M, Gong Y, Smits P, Lefebvre V, Jay GD, Stewart M, Wang H, Warman ML, Carpten JD: The secreted glycoprotein lubricin protects cartilage surfaces and inhibits synovial cell overgrowth. J Clin Invest 2005, 115:622-631.

8. Teeple E, Elsaid KA, Fleming BC, Jay GD, Aslani K, Crisco JJ, Mechrefe AP: Coefficients of friction, lubricin, and cartilage damage in the anterior cruciate ligament-deficient guinea pig knee. J Orthop Res 2008, 26:231-237.

9. Young AA, McLennan S, Smith MM, Smith SM, Cake MA, Read RA, Melrose J, Sonnabend DH, Flannery CR, Little CB: Proteoglycan 4 downregulation in a sheep meniscectomy model of early osteoarthritis. Arthritis Res Ther 2006, 8:R41.

10. Jay GD, Torres JR, Rhee DK, Helminen HJ, Hytinnen MM, Cha CJ, Elsaid K, Kim KS, Cui Y, Warman ML: Association between friction and wear in diarthrodial joints lacking lubricin. Arthritis Rheum 2007, 56:3662-3669. 
11. Flannery $C R$, Zollner $R$, Corcoran $C$, Jones $A R$, Root $A$, Rivera-Bermúdez MA, Blanchet T, Gleghorn JP, Bonassar LJ, Bendele AM, Morris EA, Glasson SS: Prevention of cartilage degeneration in a rat model of osteoarthritis by intraarticular treatment with recombinant lubricin. Arthritis Rheum 2009, 60:840-847.

12. Nugent GE, Aneloski NM, Schmidt TA, Schumacher BL, Voegtline MS, Sah RL: Dynamic shear stimulation of bovine cartilage biosynthesis of proteoglycan 4. Arthritis Rheum 2006, 54:1888-1896.

13. Nugent GE, Schmidt TA, Schumacher BL, Voegtline MS, Bae WC, Jadin KD, Sah RL: Static and dynamic compression regulate cartilage metabolism of proteoglycan 4 (PRG4). Biorheology 2006, 43:191-200.

14. Nugent-Derfus GE, Takara T, O'Neill JK, Cahill SB, Görtz S, Pong T, Inoue H, Aneloski NM, Wang WW, Vega Kl, Klein TJ, Hsieh-Bonassera ND, Bae WC, Burke JD, Bugbee WD, Sah RL: Continuous passive motion applied to whole joints stimulates chondrocyte biosynthesis of PRG4. Osteoarthritis Cartilage 2007, 15:566-574.

15. Saxon L, Finch C, Bass S: Sports participation, sports injuries and osteoarthritis: implications for prevention. Sports Med 1999, 28:123-135.

16. Chang Q, Huang C, Huang Z: Matrix metalloproteinases and inhibitor in knee synovial fluid as cartilage biomarkers in rabbits: the effect of highintensity jumping exercise. J Surg Res 2007, 140:149-157.

17. Vanwanseele $B$, Lucchinetti $E$, Stussi $E$ : The effects of immobilization on the characteristics of articular cartilage: current concepts and future directions. Osteoarthr Cartil 2002, 10:408-419.

18. Otterness IG, Eskra JD, Bliven ML: Exercise protects against articular cartilage degeneration in the hamster. Arthritis Rheum 1998, 41:2068-2076.

19. Kaiki G, Tsuji H, Yonezawa T, Sekido H, Takano T, Yamashita S, Hirano N, Sano A: Osteoarthrosis induced by intra-articular hydrogen peroxide injection and running load. J Orthop Res 1990, 8:731-740.

20. Ni GX, Zhan LQ, Gao MQ, Lei L, Zhou YZ, Pan YX: Matrix metalloproteinase-3 inhibitor retards treadmill running-induced cartilage degradation in rats. Arthritis Res Ther 2011, 13:R192.

21. Pap G, Eberhardt R, Sturmer I, Machner A, Schwarzberg H, Roessner A, Neumann W: Development of osteoarthritis in the knee joints of Wistar rats after strenuous running exercise in a running wheel by intracranial self-stimulation. Pathol Res Pract 1998, 194:41-47.

22. Wei L, Fleming BC, Sun X, Teeple E, Wu W, Jay GD, Elsaid KA, Luo J, Machan JT, Chen Q: Comparison of differential biomarkers of osteoarthritis with and without posttraumatic injury in the Hartley guinea pig model. J Orthop Res 2010, 28:900-906.

23. Bedford TG, Tipton CM, Wilson NC, Oppliger RA, Gisolfi CV: Maximum oxygen consumption of rats and its changes with various experimental procedures. J Applied Physio 1979, 47:1278-1283.

24. Lee YJ, Park JA, Yang SH, Kim KY, Kim BK, Lee EY, Lee EB, Seo JW, Echtermeyer F, Pap T, Song YW: Evaluation of osteoarthritis induced by treadmill-running exercise using the modified Mankin and the new OARSI assessment system. Rheumatol Int 2011, 31:1571-1576.

25. Lane Smith R, Trindade MC, Ikenoue T, Mohtai M, Das P, Carter DR, Goodman SB, Schurman DJ: Effects of shear stress on articular chondrocyte metabolism. Biorheology 2000, 37:95-107.

26. Leong DJ, Hardin JA, Cobelli NJ, Sun HB: Mechanotransduction and cartilage integrity. Ann NY Acad Sci 2011, 1240:32-37.

27. Galois L, Etienne S, Grossin L, Cournil C, Pinzano A, Netter P, Mainard D, Gillet P: Moderate-impact exercise is associated with decreased severity of experimental osteoarthritis in rats. Rheumatology 2003, 42:692-693.

28. Jay GD, Fleming BC, Watkins BA, McHugh KA, Anderson SC, Zhang LX, Teeple E, Waller KA, Elsaid KA: Prevention of cartilage degeneration and restoration of chondroprotection by lubricin tribosupplementation in the rat following anterior cruciate ligament transection. Arthritis Rheum 2010, 62:2382-2391.

29. Teeple E, Elsaid KA, Jay GD, Zhang L, Badger GJ, Akelman M, Bliss TF, Fleming $B C$ : Effects of supplemental intra-articular lubricin and hyaluronic acid on the progression of posttraumatic arthritis in the anterior cruciate ligament-deficient knee. Am J Sports Med 2011, 39:164-172.

30. Elsaid KA, Zhang L, Waller K, Tofte J, Teeple E, Fleming BC, Jay GD: The impact of forced joint exercise on lubricin biosynthesis from articular cartilage following ACL transection and intra-articular lubricin's effect in exercised joints following ACL transection. Osteoarthritis Cartilage 2012, 20:940-948.
31. Coles JM, Zhang L, Blum JJ, Warman ML, Jay GD, Guilak F, Zauscher S: Loss of cartilage structure, stiffness, and frictional properties in mice lacking PRG4. Arthritis Rheum 2010, 62:1666-1674.

32. Drewniak El, Jay GD, Fleming BC, Zhang L, Warman ML, Crisco JJ: Cyclic loading increases friction and changes cartilage surface integrity in lubricin-mutant mouse knees. Arthritis Rheum 2012, 64:465-473.

33. Chan SMT, Neu CP, Komvopoulos K, Reddi AH: The role of lubricant entrapment at biological interfaces: Reduction of friction and adhesion in articular cartilage. J Biomech 2011, 44:2015-2020.

34. Bugbee WD, Sah RL: Continuous Passive Motion Applied to Whole Joints Stimulates Chondrocyte Biosynthesis of PRG4. Osteoarthritis Cartilage 2007, 15:566-574.

35. Neu CP, Khalafi A, Komvopoulos K, Schmid TM, Reddi AH: Mechanotransduction of bovine articular cartilage superficial zone protein by transforming growth factor beta signaling. Arthritis Rheum 2007, 56:3706-3714.

36. Seedhom BB: Conditioning of cartilage during normal activities is an important factor in the development of osteoarthritis. Rheumatology 2006, 45:146-149.

37. Darling EM, Athanasiou KA: Growth factor impact on articular cartilage subpopulations. Cell Tissue Res 2005, 322:463-473.

38. Khalafi A, Schmid TM, Neu C, Reddi AH: Increased accumulation of superficial zone protein (SZP) in articular cartilage in response to bone morphogenetic protein-7 and growth factors. J Orthop Res 2007, 25:293-303.

39. DuRaine G, Neu CP, Chan SMT, Komvopoulos K, June RK, Reddi AH: Regulation of the friction coefficient of articular cartilage by TGF- $\beta 1$ and IL-1ß. J Orthop Res 2009, 27:249-256.

40. Jones ARC, Flannery CR: Bioregulation of lubricin expression by growth factors and cytokines. Euro Cells Mater 2007, 13:40-45.

41. Schmidt TA, Schumacher BL, Han EH, Klein TJ, Voegtline MS, Sah RL: Synthesis and secretion of lubricin/superficial zone protein by chondrocytes in cartilage explants: modulation by TGF- $\beta 1$ and IL-1a. Trans Orthop Res Soc 2004, 29:577.

doi:10.1186/ar4101

Cite this article as: Ni et al:: Intensity-dependent effect of treadmill running on lubricin metabolism of rat articular cartilage. Arthritis Research \& Therapy 2012 14:R256.

\section{Submit your next manuscript to BioMed Central and take full advantage of:}

- Convenient online submission

- Thorough peer review

- No space constraints or color figure charges

- Immediate publication on acceptance

- Inclusion in PubMed, CAS, Scopus and Google Scholar

- Research which is freely available for redistribution

Submit your manuscript at www.biomedcentral.com/submit 